\title{
A thermal quench induces spatial inhomogeneities in a holographic superconductor
}

\author{
Antonio M. García-García, ${ }^{a, b}$ Hua Bi Zeng ${ }^{b, c}$ and Hai-Qing Zhang ${ }^{b}$ \\ ${ }^{a}$ Cavendish Laboratory, University of Cambridge, \\ JJ Thomson Avenue, Cambridge, CB3 OHE, U.K. \\ ${ }^{b}$ CFIF, Instituto Superior Técnico, Universidade Técnica de Lisboa, \\ Av. Rovisco Pais, 1049-001 Lisboa, Portugal \\ ${ }^{c}$ School of Mathematics and Physics, Bohai University, \\ JinZhou 121000, China \\ E-mail: amg73@cam.ac.uk, zenghbi@gmail.com, joeycheunghq@gmail.com
}

ABSTRACT: Holographic duality is a powerful tool to investigate the far-from equilibrium dynamics of superfluids and other phases of quantum matter. For technical reasons it is usually assumed that, after a quench, the far-from equilibrium fields are still spatially uniform. Here we relax this assumption and study the time evolution of a holographic superconductor after a temperature quench but allowing spatial variations of the order parameter. Even though the initial state and the quench are spatially uniform we show the order parameter develops spatial oscillations with an amplitude that increases with time until it reaches a stationary value. The free energy of these inhomogeneous solutions is lower than that of the homogeneous ones. Therefore the former corresponds to the physical configuration that could be observed experimentally.

Keywords: Duality in Gauge Field Theories, AdS-CFT Correspondence, Black Holes

ARXiv EPRINT: 1308.5398 


\section{Contents}

1 Introduction 1

2 The model and the boundary conditions 2

3 Definition of the quench and solution of the EOM 3

4 The spatially inhomogeneous solution 4

5 Stability of the inhomogeneous solution $\quad 7$

A Vacuum Expectation Value $\langle O\rangle \quad 9$

\section{Introduction}

Most physical processes occur under non-equilibrium conditions. Small deviations from equilibrium are well understood in the framework of linear response theory. However the description of the dynamics beyond linear response is still one of the most challenging problems in theoretical physics. Recent experimental advances in the study of the farfrom equilibrium dynamics after a quench are opening new research avenues in condensed matter [1-3] and cold atom physics [4]. A typical example is the study of the spontaneous generation of defects [4] in a Bose gas after a temperature quench across the superfluid transition which is qualitatively described by the Kibble-Zurek mechanism [5-7].

More quantitative theoretical results are known [8-10] in the more tractable problem of the dynamics of a zero dimensional mean-field superconductor after a quantum quench. An analytical study [8-10] of the far-from equilibrium time evolution of a Bardeen-CooperSchrieffer (BCS) superconductor resulted, in a certain region of parameters, in undamped time oscillations of the order parameter. However it was later [11] realized that for system sizes larger than the superconducting coherence length the quench can excite finite momentum states. This results in spatial inhomogeneities of the order parameter that make the time oscillations unstable. Exact results in a one dimension quantum spin-chain that is driven from paramagnetic to ferromagnetic [12] confirm this picture.

Despite these advances there is not yet a comprehensive theoretical framework to describe quantitatively most of these phenomena. The recent introduction of the holographic principle, also called the (Anti de Sitter/Conformal field theory) AdS/CFT correspondence [13-15], in this context [16-20] has broaden considerably the theoretical tools to tackle non-equilibrium problems. In the context of holographic superconductivity [21, 22], the problem we study here, there are already several studies that employ AdS/CFT techniques to describe the time evolution of the order parameter after a thermal [23-26] or quantum [27, 28] quench. In these papers it was assumed that the order parameter was spatially uniform. This is a useful simplification since the gravity equations of motion depend only on two instead of three variables. However from the above discussion it is 
plausible that spatial inhomogeneities play a important role in the dynamic evolution of the order parameter. Indeed recent AdS/CFT calculations have shown [29-31] that a coupling to an axion field or a topological Chern-Simon term can induce a spontaneously breaking of translational invariance. Spatially inhomogeneous solutions of the gravity equations are also a crucial ingredient in the recent description of two dimensional turbulence [32] by holographic techniques. The dynamics after a soft quench across a thermal or quantum critical point suggests [34] that spatially inhomogeneous solutions might be stable. In the context of heavy ion collisions it was recently studied the far-from equilibrium dynamics in the presence of spatial inhomogeneities [33]. It is therefore timely to ask whether the spontaneous breaking of translational symmetry can also be induced by a quench. Here we respond this question affirmatively. We study the evolution of the order parameter of a holographic superconductor after a quench induced by turning on the source of the order parameter. Even though the initial state and the quench are spatially homogeneous we have observed that, for all quenches studied, the order parameter becomes spatially inhomogeneous for sufficiently long times. This spatially non-uniform solution has a lower free energy than the homogeneous one. We start our analysis by introducing the gravity dual and working out the solutions of the equations of motion (EOM).

\section{The model and the boundary conditions}

The starting action in the usual gravity dual of a holographic superconductor is [21, 22], $S=\int d^{4} x \sqrt{-g}\left[R-2 \Lambda-\frac{1}{4} F_{\mu \nu} F^{\mu \nu}-|\nabla \psi-i q A \psi|^{2}-m^{2}|\psi|^{2}\right]$ where $\Lambda=-d(d-1) / 2 L^{2}$ is the cosmological constant while $d$ is the dimension of the boundary, $F_{\mu \nu}=\partial_{\mu} A_{\nu}-$ $\partial_{\nu} A_{\mu}$ is the strength of the gauge field. The metric is an AdS Schwarzschild black hole, $d s^{2}=-f(r) d t^{2}+\frac{d r^{2}}{f(r)}+r^{2}\left(d x^{2}+d y^{2}\right)$ with $f(r)=r^{2} / L^{2}\left(1-r_{0}^{3} / r^{3}\right), r$ the bulk radial coordinate, $r_{0}$ the horizon position, and $x, y$ the boundary coordinates. Without loss of generality we set $q=1, L=1$. The temperature of the black hole is $T=\frac{3 r_{0}}{4 \pi}$.

We aim to find solutions that depend explicitly not only on time and the holographic coordinate $r$ but also on the spatial coordinate $x$ in the boundary, $\psi=\psi(t, r, x), \psi^{*}=$ $\psi^{*}(t, r, x), A=\left(A_{t}(t, r, x), A_{r}(t, r, x), A_{x}(t, r, x), 0\right)$. However these functions are not gaugeinvariant. In order to define gauge-invariant fields, we employ the following gauge transformations, $\psi(t, r, x)=\rho(t, r, x) e^{i \varphi(t, r, x)}, \psi^{*}(t, r, x)=\rho(t, r, x) e^{-i \varphi(t, r, x)}$ and $A_{i}(t, r, x)=$ $M_{i}(t, r, x)+\partial_{i} \varphi(t, r, x), \quad i=t, r, x$. The EOM resulting from the gravity Einstein equations for the gauge invariant fields $\rho$ and $M_{i}$ are,

$$
\begin{aligned}
& \frac{\partial_{x}^{2} M_{t}}{r^{2} f}-\frac{2 M_{t} \rho^{2}}{f}-\frac{\partial_{t x} M_{x}}{r^{2} f}-\frac{2 \partial_{t} M_{r}}{r}-\partial_{t r} M_{r}+\frac{2 \partial_{r} M_{t}}{r}+\partial_{r}^{2} M_{t}=0, \\
& -\frac{f \partial_{x}^{2} M_{r}}{r^{2}}+2 f M_{r} \rho^{2}+\frac{f \partial_{r x} M_{x}}{r^{2}}+\partial_{t}^{2} M_{r}-\partial_{t r} M_{t}=0, \\
& f f^{\prime} \partial_{x} M_{r}-f f^{\prime} \partial_{r} M_{x}+f^{2} \partial_{r x} M_{r}-f^{2} \partial_{r}^{2} M_{x}+2 f M_{x} \rho^{2}-\partial_{t x} M_{t}+\partial_{t}^{2} M_{x}=0, \\
& \rho\left(f\left(\frac{M_{x}^{2}}{r^{2}}+m^{2}\right)+f^{2} M_{r}^{2}-M_{t}^{2}\right)-\frac{f \partial_{x}^{2} \rho}{r^{2}}-f^{2} \partial_{r}^{2} \rho+\partial_{t}^{2} \rho-\frac{f\left(r f^{\prime}+2 f\right) \partial_{r} \rho}{r}=0, \\
& \rho\left(-r^{2} f f^{\prime} M_{r}-r^{2} f^{2} \partial_{r} M_{r}-2 r f^{2} M_{r}-f \partial_{x} M_{x}+r^{2} \partial_{t} M_{t}\right) \\
& -2 r^{2} f^{2} M_{r} \partial_{r} \rho-2 f M_{x} \partial_{x} \rho+2 r^{2} M_{t} \partial_{t} \rho=0 .
\end{aligned}
$$


We note that the phase $\varphi$ is automatically cancelled and that the last equation (2.5) is a linear combination of the first three equations, i.e., eqs. (2.1), (2.2) and (2.3). Therefore we have a well defined problem as there are four independent partial differential equations and four fields, $\rho, M_{t}, M_{r}$ and $M_{x}$.

In the limit of time independent fields, it is clear that only eq. (2.1) and (2.4) survive as $M_{r}=0$ and $M_{x}=0$ are solutions to the above equations. However time dependent solutions require to turn on $M_{r}$ and $M_{x}$ in order for the EOM to be gauge invariant and self-consistent.

We can now introduce the boundary conditions needed to solve the EOM. Following the standard AdS/CFT dictionary, close to the boundary we impose,

$$
M_{t}=\mu(t, x)-\tilde{\rho}(t, x) / r+\ldots,
$$

and $\rho=\rho_{1}(t, x) / r+\rho_{2}(t, x) / r^{2}+\ldots, M_{r}=M_{r}^{(2)}(t, x) / r^{2}+\ldots, M_{x}=v(t, x)+\tilde{J}(t, x) / r+\ldots$ where we have set $m^{2}=-2, \tilde{\rho}$ is the charge density and $\mu$ is the chemical potential. Before the quench we impose $\rho_{1}=0$ so that $\rho_{2}$ is the order parameter,

$$
\langle\mathcal{O}(x, t)\rangle \equiv \rho_{2}(x, t) .
$$

Since we do not consider the case of a finite super-current we can safely set $\tilde{J}=0$. At the horizon we impose that $M_{t}=0$ and that the rest of functions have no singularities. The next task is to define the thermal quench and to solve the EOM by a suitable numerical algorithm.

\section{Definition of the quench and solution of the EOM}

We aim to study the time evolution of the order parameter $\langle\mathcal{O}(x, t)\rangle$ after a quench, namely, an abrupt change in the system. Following ref. [24,28] we induce the quench by turning on the source in the expansion of scalar around the boundary, $\rho=\rho_{1}(t, x) / r+\rho_{2}(t, x) / r^{2}+\ldots$ with

$$
\rho_{1}=J \tanh (v t) .
$$

Qualitatively the physical situation that we want to describe is the time evolution of the order parameter of a superfluid which is suddenly put in contact with other superconductor (the source). In the condensed matter literature this is what is called superconductivity by "proximity effect". In our setting, the source, if turned on fast enough, changes abruptly the properties of the initial superfluid which induces an out of equilibrium evolution of the order parameter. The Gaussian quench employed in ref. [24] is less suitable for our purposes. The calculation of ref. [24] includes backreaction so this Gaussian quench effectively changes the temperature of the field theory despite the fact the source is turned on only for a brief period of time. We are in the probe limit so the temperature in our calculation is fixed. For that reason thy type of quench is not suitable for our purposes.

We note that with our choice of coordinates the order parameter is still given by $\rho_{2}(t, x)$, please consult the appendix for more details. For $v \ll 1$ the source goes from zero to $J$ very quickly which has the effect to excite the system to a far-from equilibrium 
state. We keep the chemical potential constant, $\mu=\mu_{0}$ and let the charge density vary. At $t=0$ we assume the system is described by a homogeneous and static solution of the EOM with $\mu(x, t=0)=\mu_{0}$. Obviously, as has been recently discussed in ref. [29-31], it is always necessary to add a tiny seed of inhomogeneity so that the numerical code can find true inhomogeneous solutions with a lower free energy. This seed is typically a (quasi)random noise of amplitude much smaller than the inhomogeneity resulting after long times after the quench. In our case it is of order $10^{-7}$ and it is induced by the spectral method that we employ. The shape and form of this initial seed is completely unrelated to the true inhomogeneities that develop for long times. Quantum and thermal fluctuations, ubiquitous in any realistic physical system, have an equivalent effect.

The EOM evolves this solution to a solution that verifies the above boundary condition for the scalar close to the boundary. We choose $v, J, \mu_{0}$ so that the system is always superfluid, namely, $\mu_{0}>\mu_{c} \propto 1 / T_{c}$ where in our quantization, $\mu_{c}=4.0636$ [21,22]. We stay relatively close to $T_{c}$ so that the probe limit that we employ is still a good approximation. In order to solve the coupled partial differential equations we used the spectral method [35]. We discretize the EOM on a three dimensional Chebyshev grid with 40 points along the $t$ direction and 20 points along the $z=1 / r$ direction, and up to 30 points along the $x$ direction. We study the time evolution for different values of $J, v$. An important comment is in order. For technical reasons we do not have much flexibility to tune these parameters. If $v$ is very large then the perturbation is very slow so it is not really a quench. Moreover it will take a long time to observe any interesting effect. For $v$ too small the perturbation is very fast, a true quench, so we expect a relatively fine structure in the time and space evolution of the order parameter which cannot be resolved by the maximum number of points that we can simulate. More specifically we need that the coherence length which controls the spatial inhomogeneities be larger than the cutoff induced by the finite lattice spacing. That constraints the values of $J \leq 1$ and $v \sim 0.1$.

\section{The spatially inhomogeneous solution}

As was mentioned previously the problem of the dynamics of a holographic superconductor after a quench has already been investigated [23-26] but in the limit of spatial homogeneity. Here we show that a thermal quench makes the order parameter spatially inhomogeneous at least for the abrupt changes of temperature that we explore in this paper. More importantly we provide compelling evidence that these solutions have a lower free energy than the homogeneous ones. Results for the quench with $J=1$ and $v=0.1$ are shown in figure 1 and figure 2. The time evolution is similar for different spatial points. However the spatial dependence strongly depends on time. For short times it is almost spatially homogeneous however, for longer times, spatial oscillations of growing amplitude are clearly observed. The dependence on $x$ seems to be oscillatory which suggests that only few Fourier modes are excited by the quench. For smaller $v$ or larger $J$ we expect a more intricate pattern. However it would require a smaller lattice spacing which is beyond our numerical capabilities. The wavevectors $k_{o}$ of the oscillations of the order parameter is inversely proportional to the superconducting coherence length $k_{o} \sim n / \xi$ with $n$ an integer. For sufficiently 

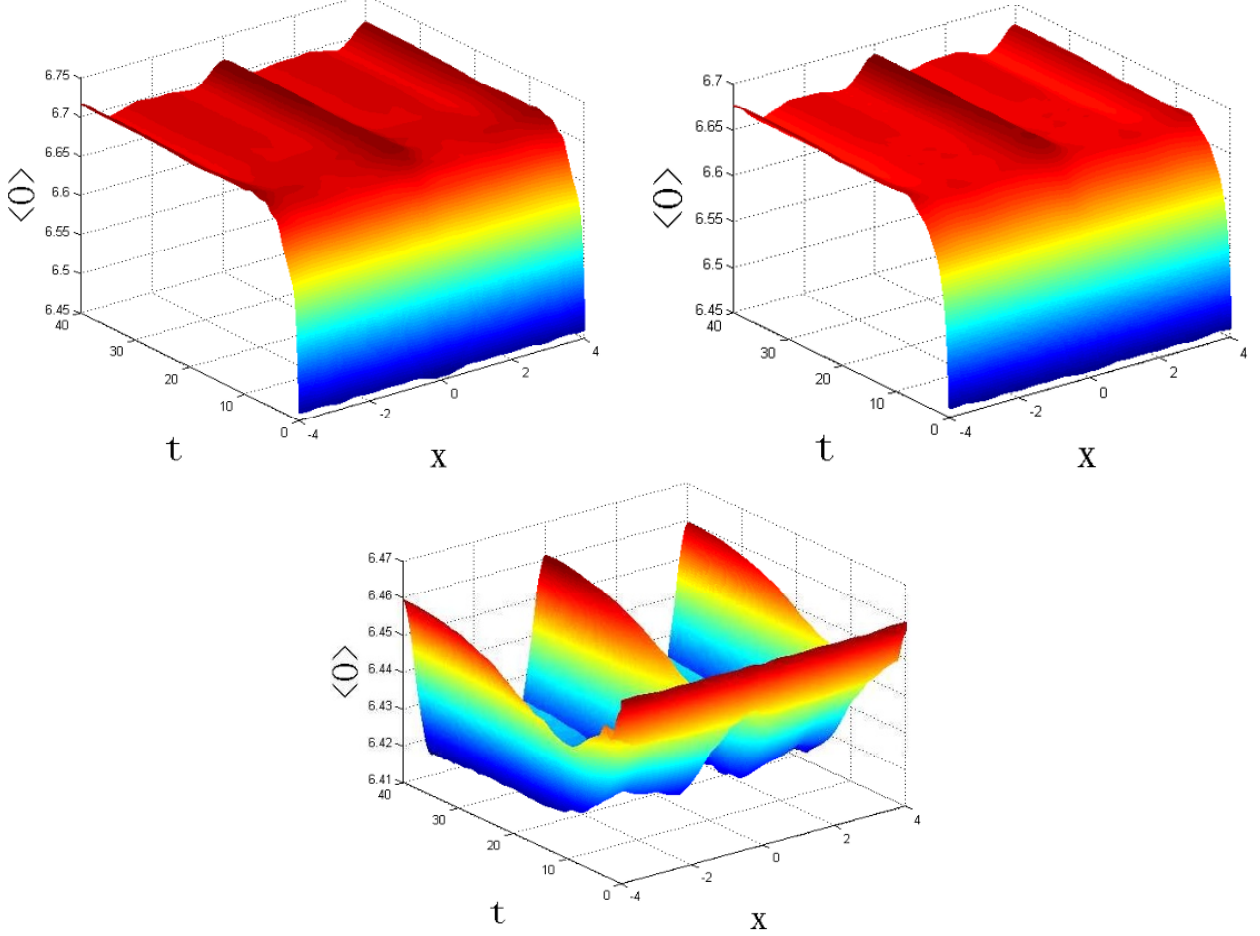

Figure 1. Space and time dependence of the the order parameter $\langle\mathcal{O}(x, t)\rangle$ eq. (2.7) after the quench eq. (3.1) with $v=0.1, \mu=6$ and, from top to bottom, $J=1.5, J=1.2$ and $J=0.1$.

strong quenches this coherence length does not have to correspond to the equilibrium one but rather to the one at which the evolution became non adiabatic [5-7]. This is nothing but a consequence of the Kibble-Zurek mechanism.

Physically this relatively simple oscillating pattern is an indication that the initially homogeneous order parameter decays into two states of finite and opposite momentum. Finally we stress that even though the temperature is well defined across the sample, namely the chemical potential $\mu$ is uniform, the order parameter $\langle\mathcal{O}\rangle$ still develops a spatial dependence that grows with time.

These findings are consistent with those previously obtained [8-10] for weakly coupled superconductors in the condensed matter literature. Physically the spatial inhomogeneities [8-10] are a consequence of re-arrangements of the order parameter in space and time after a quench which are compatible with the conservation of energy and momentum [8-10]. Similar results, depicted in figure 3, are observed for other quench parameters.

In summary in the range of parameters that we can explore numerically we observe that, after the quench eq. (3.1), the superconductor eventually becomes spatially inhomogeneous. However these results assume that the order parameter is still defined as $\langle O\rangle=\rho_{2}$ after the quench. How can be sure about it? In principle the definition of the order parameter might depend on the quench details and the system of coordinates. In the appendix we carry out the calculation explicitly for our case and show that indeed $\langle O\rangle=\rho_{2}$ after the quench. As an additional check we also verify numerically, see figure 4, that the only contribution to $\rho_{1}$ comes from the quench itself $J \tanh (v t)$. 

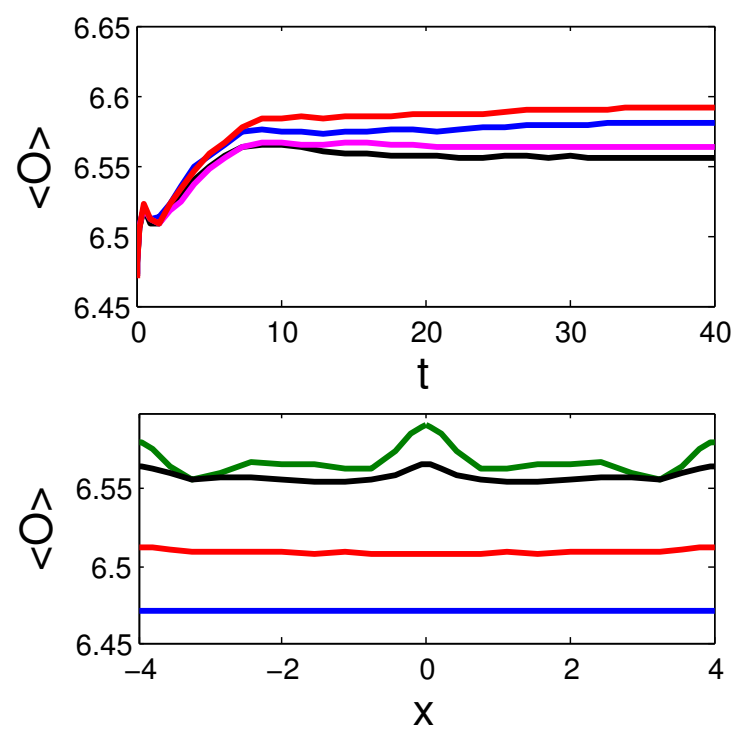

Figure 2. Upper: the order parameter $\langle\mathcal{O}(t)\rangle$ for a quench $\mu=6, v=0.1, J=1.2$ as a function of time for different $x$ 's: $x=0$ (red), $x=1.1322$ (pink), $x=3.247$ (black) and $x=4$ (blue). Lower: the order parameter $\langle\mathcal{O}(x)\rangle$, for the same quench but for different times, $t=0$ (blue), $t=1$ (red) $t=5$ (black), and $t=40$ (green).
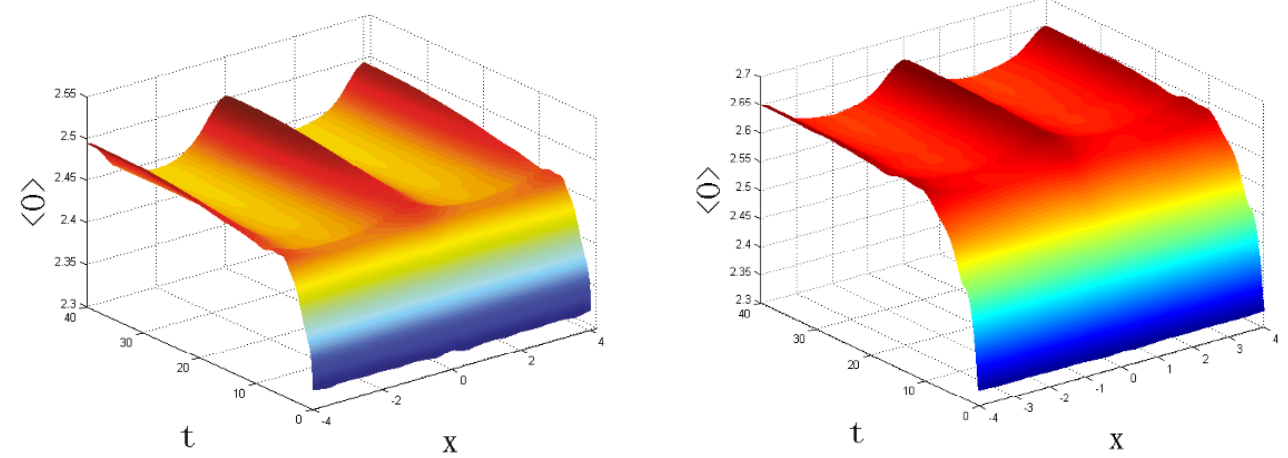

Figure 3. Space and time dependence of the the order parameter $\langle\mathcal{O}(x, t)\rangle$ eq. (2.7) after the quench eq. (3.1) with $v=0.1, \mu=4.5$ and $J=0.8$ (left), $J=1.2$ (right).

Another issue more specific of the quench eq. (3.1) is that it does not vanish for $t \rightarrow \infty$. In principle it is not clear whether the final state corresponds to a system with spontaneous symmetry breaking. Previously [36] it has been shown that in certain cases a double trace deformation, similar to adding a source, can still induce spontaneous symmetry breaking. Here our motivation is not to fully enter in this question but rather to show that our results are independent of the quench details or the exact nature of the final state. For that purpose we also study, see figure 5 , a quench $\rho_{1}=J(1-\tanh (v t))$ for which the source vanishes for sufficiently long times and therefore the symmetry is, without any doubt, spontaneously broken. As was expected the results for this quench are almost identical to those obtained from the eq. (3.1). This is a further confirmation that the spontaneous generation of spatial inhomogeneities is a universal phenomenon that do not depend on the quench details. 


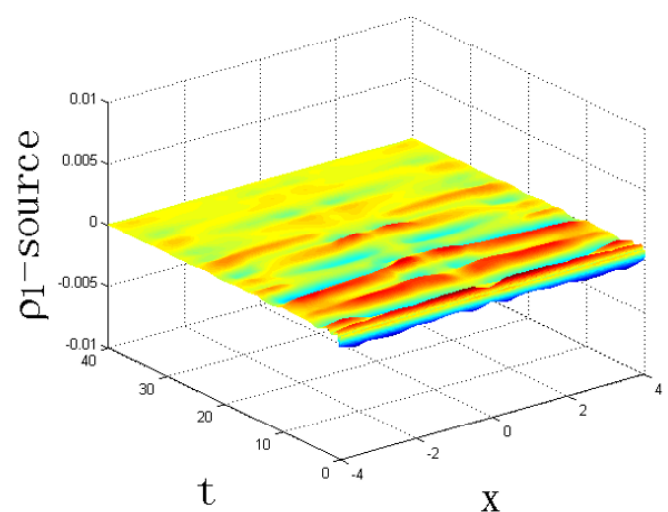

Figure 4. The difference between the numerical result of $\rho_{1}$ and the value of the source $J \tanh (v t)$ in the case $v=0.1, \mu=6$ and $J=1.5$. It is clearly observed that the difference is extremelly small for all times considered. This is a further confirmation that also after the quench $\langle O\rangle=\rho_{2}$.

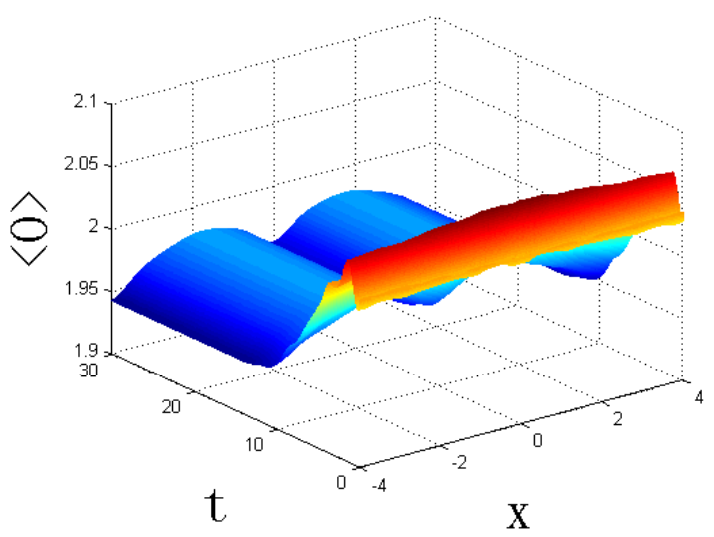

Figure 5. Space and time dependence of the the order parameter $\langle\mathcal{O}(x, t)\rangle$ eq. (2.7) after the quench $\rho_{1}=J(1-\tanh (v t))$ for $\mu=4.1, J=0.1$ and $v=1.1$.

\section{Stability of the inhomogeneous solution}

Next we investigate whether these inhomogeneous solutions correspond to the physical state of minimum energy. For that purpose we compare the free energy of the homogeneous and non-homogeneous solutions for long times around $t=t_{f}$ so that spatial inhomogeneities are more clearly observed. The free energy $F=-T S_{\mathrm{os}}+\ldots$ is directly related to the on shell action $S_{\mathrm{os}}$ [37]. In order to evaluate $S_{\mathrm{os}}$ it is convenient to integrate by parts and use the equations of motion which results in,

$S_{\mathrm{os}}=\int_{z=0} d^{3} x\left[\frac{h}{z^{2}} \rho \rho^{\prime}-M_{t} M_{t}^{\prime}+h M_{x} M_{x}^{\prime}+h^{2} M_{r} M_{r}^{\prime}\right]-\int d^{4} x\left[\frac{M_{t}^{2}}{h z^{2}} \rho^{2}-\frac{M_{x}^{2} \rho^{2}}{z^{2}}+\frac{h}{z^{2}} M_{r}^{2} \rho^{2}\right]$,

where $z=1 / r, h(z)=1-z^{3}$ and ' stands for the derivative with respect to z. We work in the grand canonical ensemble characterized by a fixed $\mu$. It is possible to show that boundary contributions have divergences coming from the scalar contribution. Fortunately this divergence can be removed by adding a counterterm. The resulting renormalized free 

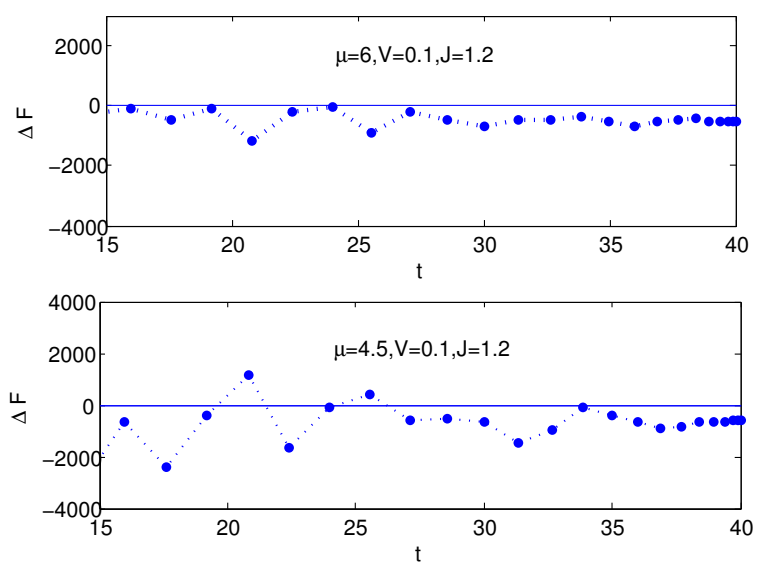

Figure 6. The difference between the free energy eq. (5.1) of the inhomogeneous and the homogeneous solution $\Delta F(t)$ as a function of time for two different quenches. Interestingly the difference becomes clearly negative after the spatial inhomogeneities become substantial. The fluctuations observed for short times are a numerical artefact. A finer grid, beyond our numerical capabilities, would be needed to capture accurately the far from equilibrium dynamic shortly after the quench.

energy is given by,

$$
F \propto \int_{z=0} d^{3} x\left[M_{t} M_{t}^{\prime}\right]+\int d^{4} x\left[\frac{M_{t}^{2}}{h z^{2}} \rho^{2}-\frac{M_{x}^{2} \rho^{2}}{z^{2}}+\frac{h}{z^{2}} M_{r}^{2} \rho^{2}\right] .
$$

This expression is already suitable to compute the free energy for both homogeneous and inhomogeneous solutions at four different times which are close to $t_{f}$. The results, depicted in figure 6, provide clear evidence that for all quenches the inhomogeneous solution has always a lower free energy. This is a confirmation that, in general, thermal quenches not only make the order parameter time dependent but also space dependent. Therefore spatial inhomogeneity is an intrinsic ingredient in the dynamics of a strongly coupled superconductor after a homogeneous thermal quench.

In conclusion we have studied the time evolution of a holographic superconductor after abruptly turning on the source of the scalar field. For all the quenches studied the solution with the lowest free energy is spatially non-uniform. Time oscillations become unstable as spatial non homogeneities develop. To a good approximation the spatial dependence is a simple oscillatory function with an amplitude that increases with time until reaches, in the range of times studied, a constant value.

\section{Acknowledgments}

AMG acknowledges illuminating conversations with Hong Liu. HQZ and HBZ thank Matthias Kaminski for fruitful discussions. AMG acknowledge support from EPSRC, grant No. EP/I004637/1, FCT, grant PTDC/FIS/111348/2009 and a Marie Curie International Reintegration Grant PIRG07-GA-2010-268172. HQZ was supported by a Marie Curie International Reintegration Grant PIRG07-GA-2010-268172. HBZ acknowledge support from FCT, grant PTDC/FIS/111348/2009, Marie Curie International Reintegration Grant PIRG07-GA-2010-268172 and the National Natural Science Foundation of China (under Grant No. 11205020). 


\section{A Vacuum Expectation Value $\langle O\rangle$}

The generic on-shell action of the Maxwell-complex scalar action is,

$$
\begin{aligned}
S_{\text {on-shell }}= & -\frac{1}{2} \int d^{4} x \partial_{a}\left[\sqrt{-g}\left(A_{b} F^{a b}+\psi^{*} \partial^{a} \psi+\psi \partial^{a} \psi^{*}\right)\right] \\
& +\frac{i q}{2} \int d^{4} x \sqrt{-g} A_{a}\left(\psi^{*} \partial^{a} \psi-\psi \partial^{a} \psi^{*}-2 i q A^{a}|\psi|^{2}\right) .
\end{aligned}
$$

The first integral in eq. (A.1) is related to the surface term since there is a total derivative before the integrand, while the second integral is in the bulk.

If we are going to calculate the vacuum expectation value (VEV) of the operator $\langle O\rangle$, we should focus on the surface integral rather than the bulk integral in eq. (A.1). It is

$$
\begin{aligned}
S_{\text {surface }}=- & \frac{1}{2} \int d^{4} x \partial_{a}\left[\sqrt{-g}\left(A_{b} F^{a b}+\psi^{*} \partial^{a} \psi+\psi \partial^{a} \psi^{*}\right)\right] \\
=-\frac{1}{2}\left[\left.\int d r d x d y r^{2}\left(-A_{r} F_{t r}-\frac{A_{x}}{f r^{2}} F_{t x}+\psi^{*} \partial^{t} \psi+\psi \partial^{t} \psi^{*}\right)\right|_{t_{i}} ^{t_{f}}\right. & \\
& +\left.\int d t d x d y r^{2}\left(-A_{t} F_{r t}+\frac{A_{x} f}{r^{2}} F_{r x}+\psi^{*} \partial^{r} \psi+\psi \partial^{r} \psi^{*}\right)\right|_{r_{0}} ^{r_{\infty}} \\
& \left.+\left.\int d t d r d y r^{2}\left(-\frac{A_{t}}{f r^{2}} F_{x t}+\frac{A_{r} f}{r^{2}} F_{x r}+\psi^{*} \partial^{x} \psi+\psi \partial^{x} \psi^{*}\right)\right|_{x_{i}} ^{x_{f}}\right] .
\end{aligned}
$$

The first and the last terms in the second equality will not be considered when we are calculating the VEV of the operator, since they are integrals in the $r$ direction which do not explicitly contain the source term $\rho_{1}$. Therefore, only the following term remains,

$$
\begin{aligned}
S_{\text {surface-new }} & =-\left.\frac{1}{2} \int d t d x d y r^{2}\left(-A_{t} F_{r t}+\frac{A_{x} f}{r^{2}} F_{r x}+\psi^{*} \partial^{r} \psi+\psi \partial^{r} \psi^{*}\right)\right|_{r_{0}} ^{r_{\infty}} \\
& =-\left.\frac{1}{2} \int d t d x d y r^{2}\left[-A_{t}\left(\partial_{r} A_{t}-\partial_{t} A_{r}\right)+\frac{A_{x} f}{r^{2}}\left(\partial_{r} A_{x}-\partial_{x} A_{r}\right)+2 f \rho \partial_{r} \rho\right]\right|_{r_{0}} ^{r_{\infty}} .
\end{aligned}
$$

Since $f\left(r_{0}\right)=A_{t}\left(r_{0}\right)=0$, therefore the above eq. (A.3) becomes,

$$
\begin{aligned}
S_{\text {surface-new }}=-\frac{1}{2} \int d t d x d y & \left.r^{2}\left(A_{t} \partial_{t} A_{r}-A_{t} \partial_{r} A_{t}\right)\right|_{r=r_{\infty}} \\
& \left.+\left.f\left(A_{x} \partial_{r} A_{x}-A_{x} \partial_{x} A_{r}\right)\right|_{r=r_{\infty}}+\left.2 f r^{2} \rho \partial_{r} \rho\right|_{r=r_{\infty}}\right] .
\end{aligned}
$$

The first two terms are finite at the infinite boundary $r=r_{\infty}$, only the last term is divergent near the boundary, therefore we can add a counter term

$$
S_{\text {counter-term }}=-\int d t d x d y \sqrt{-\gamma}|\psi|^{2}=-\int d t d x d y \sqrt{-\gamma} \rho^{2},
$$


where $\gamma$ is a reduced metric on the boundary and $\sqrt{-\gamma}=r^{2} \sqrt{f}$. Therefore, the regularized action is

$$
\begin{aligned}
S_{\text {regular }}= & S_{\text {surface }- \text { new }}+S_{\text {counter-term }} \\
= & -\left.\frac{1}{2} \int d t d x d y\left[r^{2}\left(A_{t} \partial_{t} A_{r}-A_{t} \partial_{r} A_{t}\right)+f\left(A_{x} \partial_{r} A_{x}-A_{x} \partial_{x} A_{r}\right)\right]\right|_{r=r_{\infty}} \\
& +\int d t d x d y \rho_{1} \rho_{2}+\mathcal{O}(1 / r) .
\end{aligned}
$$

Therefore, the VEV $\langle O\rangle$ is

$$
\langle O\rangle=\frac{\delta S_{\text {regular }}}{\delta \rho_{1}}=\rho_{2}
$$

which is the same as the one in ref. [21, 22].

Open Access. This article is distributed under the terms of the Creative Commons Attribution License (CC-BY 4.0), which permits any use, distribution and reproduction in any medium, provided the original author(s) and source are credited.

\section{References}

[1] C.L. Smallwood et al., Tracking Cooper Pairs in a Cuprate Superconductor by Ultrafast Angle-Resolved Photoemission, Science 336 (2012) 1137 [arXiv: 1206. 2300].

[2] T. Rohwer et al., Collapse of long-range charge order tracked by time-resolved photoemission at high momenta, Nature 471 (2011) 490.

[3] D. Fausti et al., Light-Induced Superconductivity in a Stripe-Ordered Cuprate, Science 331 (2011) 189.

[4] C.N. Weiler et al., Spontaneous vortices in the formation of Bose-Einstein condensates, Nature 455 (2008) 948 [arXiv:0807.3323].

[5] T.W.B. Kibble, Topology of Cosmic Domains and Strings, J. Phys. A 9 (1976) 1387 [INSPIRE].

[6] W.H. Zurek, Cosmological Experiments in Superfluid Helium?, Nature 317 (1985) 505 [INSPIRE].

[7] P. Laguna and W.H. Zurek, Density of kinks after a quench: When symmetry breaks, how big are the pieces?, Phys. Rev. Lett. 78 (1997) 2519 [gr-qc/9607041] [INSPIRE].

[8] R.A. Barankov and L.S. Levitov, Synchronization in the BCS Pairing Dynamics as a Critical Phenomenon, Phys. Rev. Lett. 96 (2006) 230403 [cond-mat/0603317].

[9] E.A. Yuzbashyan and M. Dzero, Dynamical Vanishing of the Order Parameter in a Fermionic Condensate, Phys. Rev. Lett. 96 (2006) 230404 [cond-mat/0603404].

[10] R.A. Barankov, L.S. Levitov and B.Z. Spivak, Collective Rabi Oscillations and Solitons in a Time-Dependent BCS Pairing Problem, Phys. Rev. Lett. 93 (2004) 160401 [cond-mat/0312053].

[11] M. Dzero, E.A. Yuzbashyan and B.L. Altshuler, Cooper pair turbulence in atomic Fermi gases, Eur. Phys. Lett. 85 (2009) 20004. [arXiv:0805.2798]. 
[12] J. Dziarmaga, Dynamics of a Quantum Phase Transition: Exact Solution of the Quantum Ising Model, Phys. Rev. Lett. 95 (2005) 245701.

[13] J.M. Maldacena, The large- $N$ limit of superconformal field theories and supergravity, Adv. Theor. Math. Phys. 2 (1998) 231 [Int. J. Theor. Phys. 38 (1999) 1113] [hep-th/9711200] [INSPIRE].

[14] S.S. Gubser, I.R. Klebanov and A.M. Polyakov, Gauge theory correlators from noncritical string theory, Phys. Lett. B 428 (1998) 105 [hep-th/9802109] [INSPIRE].

[15] E. Witten, Anti-de Sitter space and holography, Adv. Theor. Math. Phys. 2 (1998) 253 [hep-th/9802150] [INSPIRE].

[16] D.T. Son and A.O. Starinets, Minkowski space correlators in AdS/CFT correspondence: Recipe and applications, JHEP 09 (2002) 042 [hep-th/0205051] [INSPIRE].

[17] P.M. Chesler and L.G. Yaffe, Horizon Formation and Far-from-Equilibrium Isotropization in a Supersymmetric Yang-Mills Plasma, Phys. Rev. Lett. 102211601 (2009) [arXiv: 0812.2053] [INSPIRE].

[18] V. Balasubramanian et al., Thermalization of Strongly Coupled Field Theories, Phys. Rev. Lett. 106191601 (2011) [arXiv: 1012.4753] [INSPIRE].

[19] P. Bizon and A. Rostworowski, On weakly turbulent instability of anti-de Sitter space, Phys. Rev. Lett. 107 (2011) 031102 [arXiv:1104.3702] [INSPIRE].

[20] O.J.C. Dias, G.T. Horowitz, D. Marolf and J.E. Santos, On the Nonlinear Stability of Asymptotically Anti-de Sitter Solutions, Class. Quant. Grav. 29 (2012) 235019 [arXiv: 1208.5772] [INSPIRE].

[21] S.S. Gubser, Breaking an Abelian gauge symmetry near a black hole horizon, Phys. Rev. D 78 (2008) 065034 [arXiv:0801.2977] [INSPIRE].

[22] S.A. Hartnoll, C.P. Herzog and G.T. Horowitz, Building a Holographic Superconductor, Phys. Rev. Lett. 101 (2008) 031601 [arXiv:0803.3295] [INSPIRE].

[23] K. Murata, S. Kinoshita and N. Tanahashi, Non-equilibrium Condensation Process in a Holographic Superconductor, JHEP 07 (2010) 050 [arXiv: 1005.0633] [INSPIRE].

[24] M.J. Bhaseen, J.P. Gauntlett, B.D. Simons, J. Sonner and T. Wiseman, Holographic Superfluids and the Dynamics of Symmetry Breaking, Phys. Rev. Lett. 110 (2013) 015301 [arXiv: 1207.4194] [INSPIRE].

[25] I. Amado, M. Kaminski and K. Landsteiner, Hydrodynamics of Holographic Superconductors, JHEP 05 (2009) 021 [arXiv:0903.2209] [INSPIRE].

[26] K. Maeda, M. Natsuume and T. Okamura, Universality class of holographic superconductors, Phys. Rev. D 79 (2009) 126004 [arXiv:0904.1914] [InSPIRE].

[27] X. Gao, A.M. Garcia-Garcia, H.B. Zeng and H.-Q. Zhang, Normal modes and time evolution of a holographic superconductor after a quantum quench, JHEP 06 (2014) 019 [arXiv: 1212.1049] [INSPIRE].

[28] P. Basu, D. Das, S.R. Das and T. Nishioka, Quantum Quench Across a Zero Temperature Holographic Superfluid Transition, JHEP 03 (2013) 146 [arXiv:1211.7076] [InSPIRE].

[29] M. Rozali, D. Smyth, E. Sorkin and J.B. Stang, Holographic Stripes, Phys. Rev. Lett. 110 (2013) 201603 [arXiv:1211.5600] [INSPIRE]. 
[30] A. Donos and J.P. Gauntlett, Holographic striped phases, JHEP 08 (2011) 140 [arXiv: 1106.2004] [INSPIRE].

[31] H. Liu, H. Ooguri, B. Stoica and N. Yunes, Spontaneous Generation of Angular Momentum in Holographic Theories, Phys. Rev. Lett. 110 (2013) 211601 [arXiv:1212.3666] [INSPIRE].

[32] A. Adams, P.M. Chesler and H. Liu, Holographic Vortex Liquids and Superfluid Turbulence, Science $\mathbf{3 4 1}$ (26 July 2013) 368 [arXiv:1212.0281] [INSPIRE].

[33] V. Balasubramanian et al., Inhomogeneous holographic thermalization, JHEP 10 (2013) 082 [arXiv: 1307.7086] [INSPIRE].

[34] P. Basu, D. Das, S.R. Das and K. Sengupta, Quantum Quench and Double Trace Couplings, JHEP 12 (2013) 070 [arXiv: 1308.4061] [INSPIRE].

[35] L.N. Trefethen, Spectral methods in MATLAB, SIAM, Philadelphia, (2000).

[36] T. Faulkner, G.T. Horowitz and M.M. Roberts, Holographic quantum criticality from multi-trace deformations, JHEP 04 (2011) 051 [arXiv: 1008.1581] [INSPIRE].

[37] K. Skenderis, Lecture notes on holographic renormalization, Class. Quant. Grav. 19 (2002) 5849 [hep-th/0209067] [INSPIRE]. 